(C) 2019 IEEE

IEEE Journal of Emerging and Selected Topics in Power Electronics, pp. 1-1, 2019

\title{
Stability Analysis of Multi-Port MVDC Distribution Networks for All-Electric Ships
}

U. Javaid, F. D. Freijedo, W. van der Merwe, et al.

This material is posted here with permission of the IEEE. Such permission of the IEEE does not in any way imply IEEE endorsement of any of EPFL's products or services. Internal or personal use of this material is permitted. However, permission to reprint / republish this material for advertising or promotional purposes or for creating new collective works for resale or redistribution must be obtained from the IEEE by writing to pubs-permissions@ieee. org. By choosing to view this document, you agree to all provisions of the copyright laws protecting it. 


\title{
Stability Analysis of Multi-Port MVDC Distribution Networks for All-Electric Ships
}

\author{
Uzair Javaid, Member, IEEE, Francisco D. Freijedo, Senior Member, IEEE, \\ Wim van der Merwe, Senior Member, IEEE, and Drazen Dujic, Senior Member, IEEE
}

\begin{abstract}
Advances in the power electronics technologies, over the years, have opened up possibilities to consider mediumvoltage dc (MVDC) distribution network as a possible evolution of the existing medium-voltage ac (MVAC) distribution networks on large ships. MVDC distribution networks provide the possibilities to increase fuel efficiency and remove bulky transformers. However, to implement industrial scale MVDC distribution networks, some critical challenges exist, such as lack of standardised equipment, system-level stability, etc. This work studies the feasibility and stability of MVDC distribution networks when a distributed layout is considered. Due to the nature of the network, a multi input multiple output (MIMO) impedance stability approach is employed for modeling and assessment. The different components are modeled according to the existing industrial medium voltage technologies. The theoretical analysis is verified by full model time domain simulations. As a summary of the contribution, the main features of the proposed study are i) realistic identification of feasibility limits, ii) definition of design rules for capacitance sizing and best placement and iii) evaluation of distances for de distribution cables and their corresponding inductances.
\end{abstract}

Index Terms-MVDC systems, stability criteria.

\section{INTRODUCTION}

$\mathbf{K}$ EY manufacturers have introduced low voltage dc (LVDC) distribution networks, in the range of 1 $20 \mathrm{MW}$, in electric ships [1]-[3]. Benefits of the dc distribution networks developed by the available low voltage (LV) power electronic technologies, e.g., rectifiers, inverters, LVDC breakers, battery storage, etc., include increased fuel efficiency, while, also removing the ac switchboards, transformers and need for synchronization among generators [1], [2], [4].

In case of larger vessels, e.g., military vessels, liquefied natural gas (LNG) tankers, drill ships, cargo ships etc., the power requirement is higher than $20 \mathrm{MW}$ and can reach as high as $100 \mathrm{MW}$ [5]-[11]. For such power levels, the LVDC distribution networks would be required to handle very large currents, which could be technically challenging due to thermal, insulation and mechanical limits of the LV equipment.

Uzair Javaid is with G\&PQS, ABB Switzerland Ltd, Austrasse, CH-5300, Turgi, Switzerland (e-mail: uzair.javaid@ch.abb.com)

Francisco D. Freijedo was with the Power Electronics Laboratory, Ecole Polytechnique Federale de Lausanne, CH-1005, Lausanne, Switzerland. (e-mail: francisco.freijedo@ieee.org)

Wim van der Merwe is with Medium Voltage Drives, ABB Switzerland Ltd, Austrasse, CH-5300, Turgi, Switzerland (e-mail: wim.van-dermerwe@ch.abb.com).

Drazen Dujic is with the Power Electronics Laboratory, Ecole Polytechnique Federale de Lausanne, CH-1005, Lausanne, Switzerland. (e-mail: drazen.dujic@epfl.ch.)
Therefore, higher voltage level dc distribution networks are needed to exploit the benefits of the dc distribution. This opens an opportunity to explore and develop medium voltage dc (MVDC) distribution networks [12], [13].

Besides the benefits of the dc distribution networks, a large number of challenges are associated with new MVDC distribution networks. These challenges include lack of standalone MVDC technologies, e.g., rectifiers, inverters, MVDC breakers, commercial MVDC cables, active and passive filters, etc. Furthermore, system level stability [14]-[19] and protection/coordination [20], [21] are two practical bottlenecks for the existence of the future dc distribution networks on ships. Both issues are related to the MVDC distribution network layout and require detailed system level studies during early design phase.

The electrical layout, for large dc ships, offers high flexibility to place large sources and loads [17]. Fig. 1 shows the two main strategies employed in the design of dc ships: centralized and distributed layouts [21]. In a centralized layout, ac cables are employed to connect generator-rectifier and inverter-load subsystems; then, all dc terminals are connected to a common dc bus through bus-bars in the cabinet. Alternatively, dc cables are used to integrate the converters into the dc distribution cabinet in the distributed layout. The distributed layout allows for installing the power converters next to the electrical machines and can achieve higher efficiency by using the dc cables which have lower power losses than that of the ac cables. In both cases, on ac-sides existing ac circuit breakers can be used; whereas, for the dc distribution, current limiting control for power electronic converters or specialized commercial dc protection equipment will be required and developed to limit and interrupt fault currents [21].

The stability related challenge, in the dc distribution networks, is mostly caused by the constant power load (CPL) behavior of the tightly regulated high power drives [16]. The different stability related issues, their analyses and possible solutions have been reported in literature [17]-[19], [22]-[33]. Among these works, [17]-[19], [22], [23] report impedance modeling of dc-side of power electronic converters and highlight the suitability of small signal analysis to linearize power electronic converters. It is also shown here that the utilization of impedance stability criteria, with the help of Nyquist diagrams or Bode plots, can help in assessing the relative stability of the de distribution networks. This methodology is further extended to multi-terminal dc distribution networks in [24]-[26]. Additionally, literature also reports mathematical 


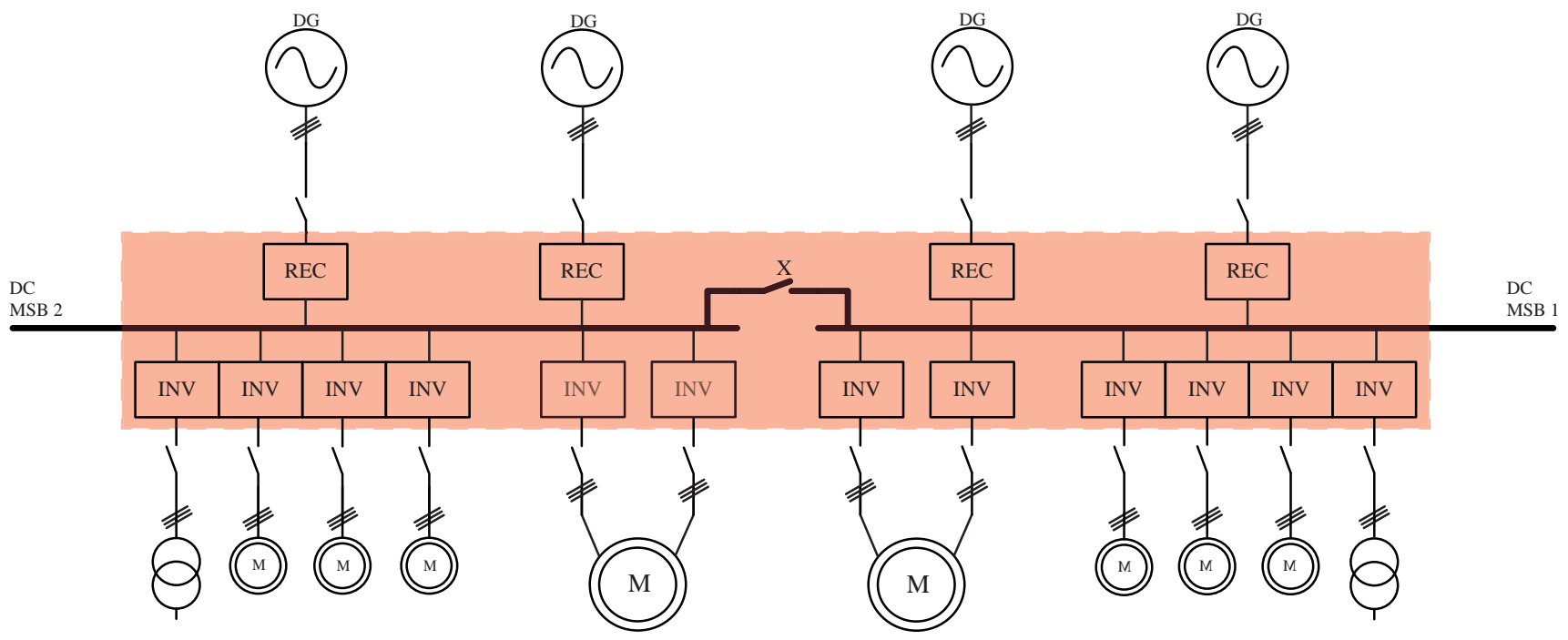

(a)

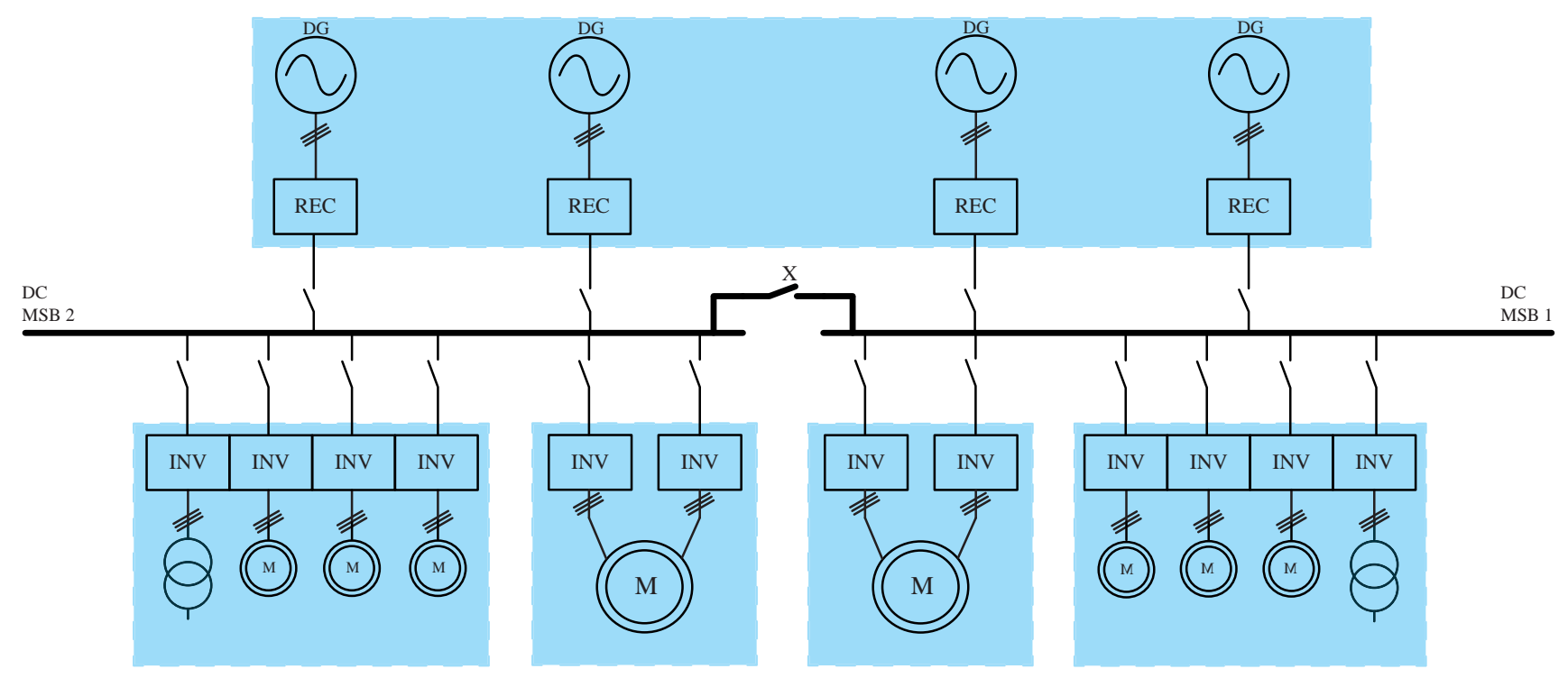

(b)

Fig. 1. Different layouts of dc distribution networks on ships. (a) Centralized - long ac cables are used to connect ac generation and load to the central platform for converters. (b) Distributed - long dc cables are used to connect the output of source converters to the load converters.

models for dc distribution networks considering continuous time and discrete time modeling techniques, which can also be used to analyze system stability [27], [28]. In addition to the stability analysis, stabilizing control techniques considering, e.g., linerization via feedback, backstepping, linear quadratic Gaussian, synergetic control, active stabilizer functionality, to name a few, are also reported in literature [29]-[33]. However, most of these reported works focus on LV converters and LVDC distribution networks.

This work addresses mutual interactions of multiple sources and loads (based on available commercial technologies) present in multi-terminal MVDC distribution networks, the impact of component placement and sizing and layout of the MVDC distribution networks for ships using multiple input multiple output (MIMO) impedance stability criteria. Impedance modeling and criteria are suitable to identify design limitations and circuit resonances [17], [34]. This study is focused on the distributed layout, which is a multi-terminal dc distribution network ${ }^{1}$. The components of the network are modeled according to the existing MVDC technologies. The results of this study identify feasibility limits and needs in terms of capacitive storage effort, best placement for capacitors and impact of the cable lengths used for the electrical distribution.

${ }^{1}$ Source and loads of a centralized distribution network can be lumped in an equivalent two-terminal network and analyzed by single-input single-output (SISO) methods. Impedance stability of two terminal MVDC distribution network for ships is already documented in [17]. 
A MIMO impedance stability approach is followed to model and assess system stability. A main reason for the choice of multivariable technique, instead of a simpler SISO approach of [7], is the fact that MIMO approach provides a complete description (observability) of the system dynamics [35], [36]. MIMO impedance/admittance transfer functions accuratelly model control actions, e.g., this approach can model system delays and frequency-dependent controllers as a part of impedance/admittance matrices [23]. It also permits to work with experimentally obtained data [37].

The rest of the paper is organized as follows. Section II explains the methodology employed for modeling and stability analyses. Section III introduces two representative case studies of 'distributed' MVDC distribution networks. Section IV provides a comprehensive frequency domain analysis for the benchmark MVDC distribution networks. The study implies a sensitivity analysis that sweeps the key parameters that affect the system stability and permits to identify feasible values for them. Subsequently, the frequency domain analysis is verified by full-model time domain simulations, in Section V. At the end, the main findings of the paper are briefly discussed in the conclusions.

\section{Multi-Port Modeling And Stability}

In this section, a brief description of the methodologies for the modeling and dynamic assessments are presented.

\section{A. Identification of Impedance of Passive Network}

The passive network consists of all the filter capacitors at the output and input of rectifiers and inverters and the cables connecting these different subsystems. Different cables/transmission line models are available in literature [38], however, $\pi$-section model ${ }^{2}$ (the simplest among them) is fairly accurate to model short cables (order of $10 \mathrm{~km}$ ). When connecting medium voltage power electronic systems with fairly modest switching frequencies (order of a few hundreds hertz) and not high $d v / d t$, a single $\pi$-section can represent a frequency up to $7 \mathrm{kHz}$ for cable lengths up to $5 \mathrm{~km}$. This can be computed by $f_{\max }=N \nu / 8 l$, where $f_{\max }$ is the maximum representable frequency, $N$ is the number of $\pi$ sections required, $\nu$ is the speed of propagation of the wave, and $l$ is the total length of the cable [38].

The portion of the MVDC distribution network composed only by passive parts can be modeled as a state-space model.

$$
\begin{aligned}
\frac{d \vec{x}(t)}{d t} & =\mathbf{A} \vec{x}(t)+\mathbf{B} \vec{u}(t) \\
\vec{y}(t) & =\mathbf{C} \vec{x}(t)
\end{aligned}
$$

Here $\vec{x}(t)$ is the state vector, $\vec{u}(t)$ is the input vector, $\vec{y}(t)$ is the output vector, $\mathbf{A}$ is the state matrix, $\mathbf{B}$ is the input matrix and $\mathbf{C}$ is the output matrix [39]. The states of the system are defined by inductance currents and capacitor voltages. The state-space model is arranged in a way that $\vec{u}(t)=\vec{i}_{d c}(t)$ is a vector of input currents defined at each capacitor. The output matrix $\mathbf{C}$ defines $\vec{y}(t)=\vec{u}_{d c}(t)$ as a vector with

\footnotetext{
${ }^{2}$ For simplicity of the analysis, it is assumed to that cables are single pole and spatially displaced
}

capacitor voltages. Following this modeling approach, the input-to-output transfer function defines an impedance matrix that is obtained by

$$
\mathbf{Z}(s)=\mathbf{C}(s \mathbf{I}-\mathbf{A})^{-1} \mathbf{B} .
$$

with

$$
\vec{V}_{d c}(s)=\mathbf{Z}(s) \vec{I}_{d c}(s)
$$

\section{B. Admittance for Active Components}

Contrary to the passive components, the active components, i.e., the converters that implement a closed loop operation, define an admittance matrix that provides the capacitor currents (i.e., the control action) as a function of the capacitor voltage measurement (i.e., controlled variables).

$$
\vec{I}_{d c}(s)=\mathbf{Y}(s) \vec{V}_{d c}(t) \text {. }
$$

Since each converter is connected in parallel to a capacitor that defines a system state, the admittance matrix can be arranged as diagonal matrix. The shape/formulation of each component of the admittance matrix depends on their mode of operation, with two main operation modes: rectifier (i.e., MVDC source) and constant power load (CPL).

For a standard rectifier, with a cascaded current and voltage control, the frequency domain behavior is dominated by the slow voltage control from this control structure $[17]^{3}$. This is mainly due to the characteristic low frequency resonance, which can cause system instability if this control is not properly tuned. The tuning of voltage controller proportional and integral gains is based on its bandwidth (which takes into account the impact of switching and sampling delays and is usually two orders of magnitude lower than the sampling frequency) and the size of capacitive filter at the terminals of the rectifier [17], [40]. Now considering that the current control has sufficient bandwidth (at least equal to the CPL one), the admittance of the rectifier would be approximated by its voltage control loop which determines the dc-side current injection into the filter capacitor and network:

$$
Y_{S}(s)=\frac{s K_{p v}+K_{i v}}{s} \times K_{c o m p}
$$

Here $K_{p v}$ and $K_{i v}$ are the proportional and integral gains of the voltage control, tuned using direct voltage control (DVC) [40], given in Fig. 2.(a), and $K_{\text {comp }}=3 V_{d} / 2 V_{d c} \approx \sqrt{3} / 2$ is the compensation factor for the current control loop, considering zero reactive power. Here possibly, if more than one rectifiers are connected to the same bus, the integral part of the control can be removed and independent droop (classic or frequency dependent) controllers can be added to the existing control [23] (this is out of the scope of the present work). In any case, irrespectively from the complexity of the controller, the relation between measured variable $v_{d c}(t)$ and control variable $i_{d c}(t)$ sets the admittance component.

On the other hand, the inverter drives, using vector control techniques, e.g., field oriented control given in Fig. 2.(b), are regulating the speed and torque of the machines and are

\footnotetext{
${ }^{3}$ Impedance characterisitics obtained in [17] are with simulation based impedance measurment.
} 


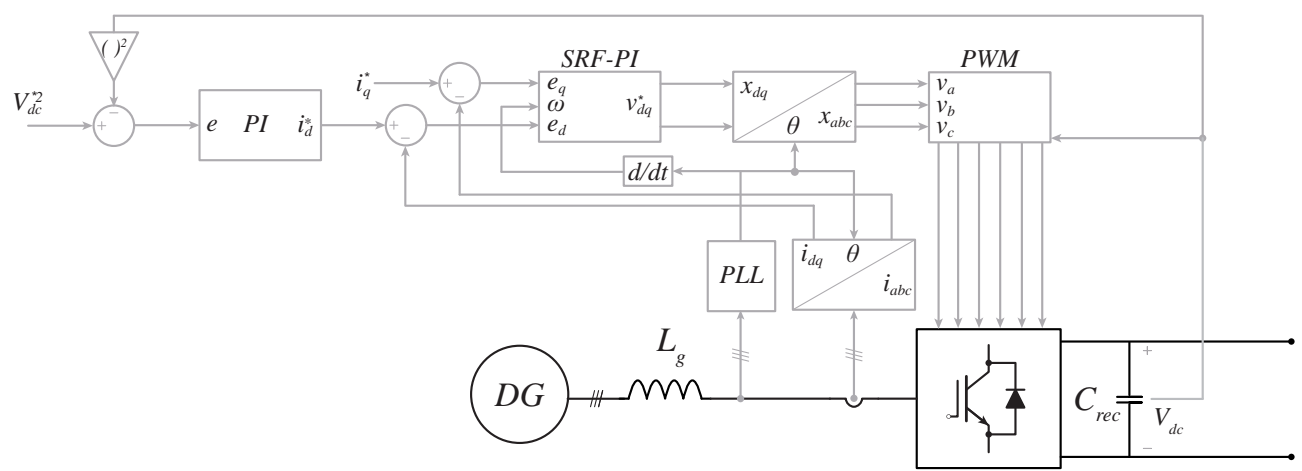

(a)

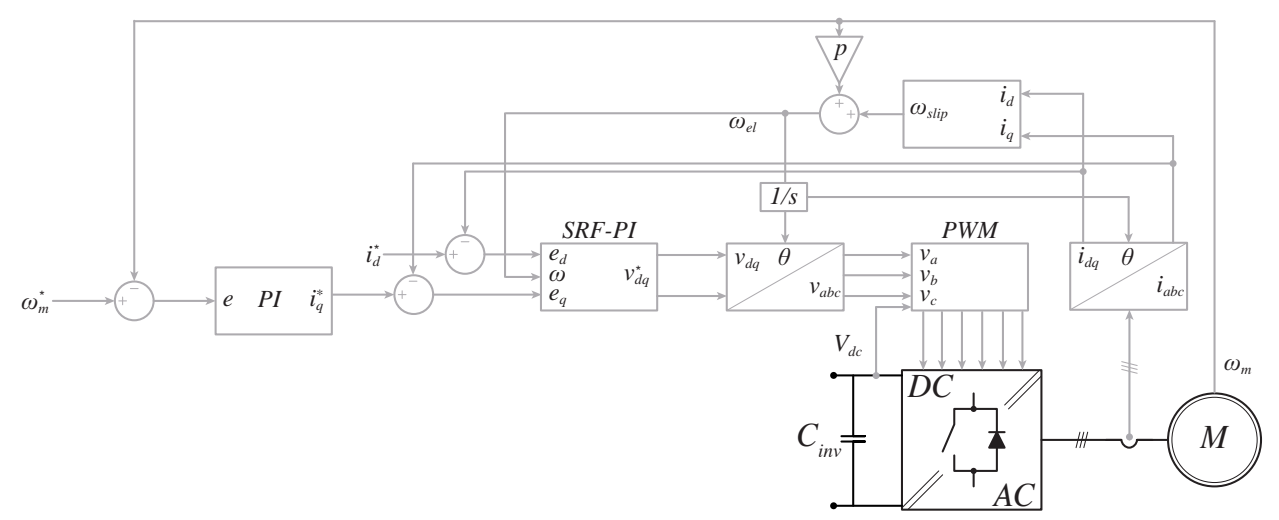

(b)

Fig. 2. Schematics of (a) active rectifier with DVC for dc-side voltage control, (b) FOC of inverter drive.

maintaining constant power for any given loading condition. Thus they are working as a CPL within their current control bandwidths or negative resistances in frequency domain [17]. This bandwidth limited behavior can be modeled by:

$$
Y_{L}(s)=-\frac{P}{V^{2}} \frac{1}{s \tau+1}
$$

with $P, V$ and $\tau$ being the load consumption power, the dc voltage (operation point), and the current control bandwidth [17], [39].

By inspection of (5) and (6), the fundamental difference between rectifier and CPL mode is the sign of the admittance on the dc-side. This has an important physical meaning: the voltage source introduces damping into the system (i.e., positive damping), but the CPL can lead it to cause instability (i.e., negative damping [17], [29], [39]). From (5), it is implicitly assumed the power supplies have an equal or larger control bandwidth than the bandwidth of CPLs, which is expected in practice; otherwise, the power supplies would have little effect on mitigating negative resistance effects, and hence, to stabilize the system.

\section{Stability Criteria for MIMO Systems}

From a linear multivariable system description, two main methods are considered in this work: i) eigenvalue analysis and ii) Nyquist criterion [41]. The eigenvalue analysis assures stability if all the eigenvalues are in the left half plane (LHP) [41]. The eigenvalues of the MIMO system are calculated by

$$
\operatorname{det}[\mathbf{I}+\mathbf{Z}(s) \mathbf{Y}(s)]=0
$$

A detailed analysis of eigenvalue movements, like participation factor and parametric sensitivities can be carried out to further exploit eigenvalue analysis [23]. However, Nyquist criterion is explored as an alternative analysis method as it is easy to use as a design tool [34]. Here the MIMO Nyquist trajectories are plotted. This helps representation of dominant poles more effectively, while avoiding poles at origin and also help in determining if there are limit cycles present in the system [42]. The Nyquist trajectories are obtained from $\operatorname{eig}[\mathbf{Z}(j \omega) \mathbf{Y}(j \omega)]$ for a given range of frequencies (starting at dc). In practice, it is possible to assume that both $\mathbf{Z}(j \omega)$ and $\mathbf{Y}(j \omega)$ do not have right half plane (RHP) zeros, so stability is assured if there are no Nyquist trajectories that encircle the citical point $(-1,0)$ [41].

\section{REPRESENTATIVE MVDC Distribution NETWORKS}

In order to provide a relatively simple but insightful study, three-port MVDC distribution networks are considered. The goal is to gradually add complexity to the simplest two-port studies (e.g., cf. [17]) by adding specific components of key relevance in an on-board MVDC distribution network. Two 


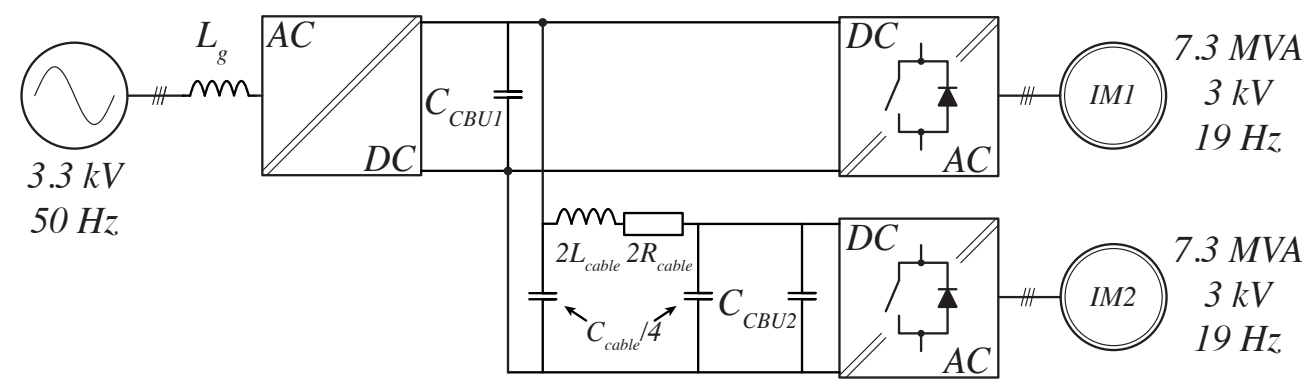

(a)

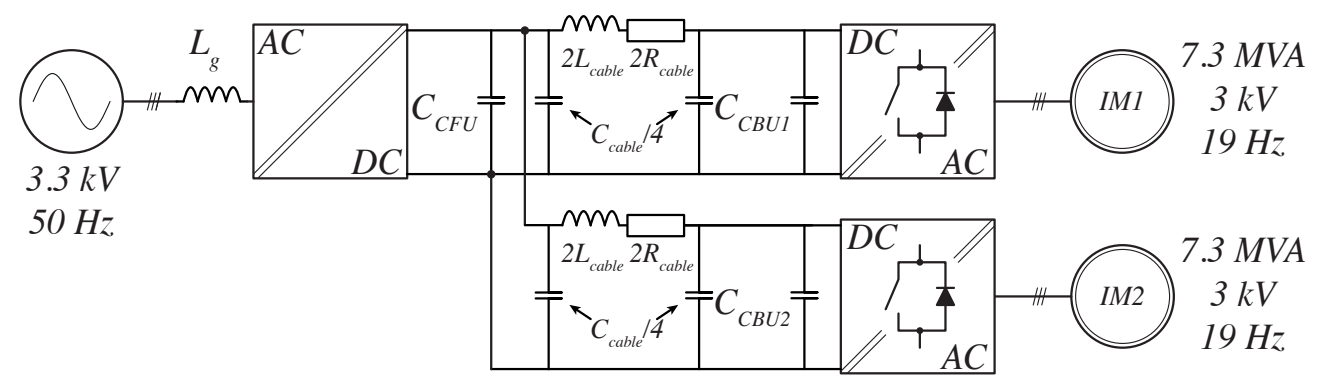

(b)

Fig. 3. Multi-terminal MVDC distribution networks. (a) Case 1. (b) Case 2.

multi-terminal MVDC distribution network layouts, depicted in Fig. 3, are considered ${ }^{4}$ :

- Case 1: One drive is connected to the source converter through the main dc-link and the second one is connected through a distribution cable. This is a special case in which one drive is located very close to the source (rectifier) and can be connected to it using short busbars. The supply is a $3.3 \mathrm{kV}, 50 \mathrm{~Hz}$ ac source connected to a rectifier through a phase reactor. The two drives are rated at 7.3 MVA each. The first drive is connected to the main capacitor bank $C B U 1$, while, the second drive is connected through a cable and has another capacitor bank $C B U 2$.

- Case 2: Both drives are connected to the source converter through distribution cables to the main dc-link. This is a more generalized version of Case 1 and a more distributed layout. This leads to the consideration of an additional capacitance bank in the system and the three capacitor banks are named $C F U, C B U 1$ and $C B U 2$. $C F U$ is the filter capacitance of the rectifier unit, while $C B U 1$ and $C B U 2$ are input filters for the inverter drives.

\section{A. Case 1 - One Drive Connected Remotely}

As explained in Section II, the impedance is derived from the passive network (cf. Fig. 4) and the admittance from the active components (closed loop controlled power electronic converters). The variables defining the passive MVDC distribution network are: (i) $i_{l}$ the current through the cable inductance, (ii) $v_{1}$ the voltage of $C_{1}$, where $C_{1}=C B U 1+$

${ }^{4}$ For simplicity, no auxiliary power sources, storage elements or loads are considered here.
TABLE I

System Parameters

\begin{tabular}{|lc|}
\hline MVDC Bus Rated Voltage & $5 \mathrm{kV}$ \\
Source Apparent Power & $14.6 \mathrm{MVA}$ \\
Source Reactance & $0.1 \mathrm{p.u}$. \\
Source Resistance & $0.01 \mathrm{p.u}$. \\
CPLs Rated Power & $6.9 \mathrm{MW}$ \\
Cable Inductance & $0.347 \mathrm{mH} / \mathrm{km}$ \\
Cable Resistance & $0.089 \Omega / \mathrm{km}$ \\
Cable Capacitance & $0.307 \mu \mathrm{F} / \mathrm{km}$ \\
Source SG Rated Frequency & $50 \mathrm{~Hz}$ \\
Load IM Rated Frequency & $19 \mathrm{~Hz}$ \\
IM Rated MVAC Voltage & $3.05 \mathrm{kV}$ \\
IM Phase Reactor Inductance & $0.0025 \mathrm{p.u}$. \\
IM Phase Reactor Resistance & $0.0025 \mathrm{p} . \mathrm{u}$. \\
IM Rated Current & $1.4 \mathrm{kA}$ \\
IM pf & 0.92 \\
VSC Switching Frequency & $f_{\text {sw }}=250 \mathrm{~Hz}$ \\
\hline
\end{tabular}

$C_{\text {cable }} / 4$, and (iii) $v_{2}$ the voltage of $C_{2}$, where $C_{2}=C B U 2+$ $C_{\text {cable }} / 4$. The state-space representation is

$$
\begin{aligned}
& x=\left[\begin{array}{l}
i_{1} \\
v_{1} \\
v_{2}
\end{array}\right], \mathbf{A}=\left[\begin{array}{ccc}
-\frac{R}{L} & \frac{1}{L} & -\frac{1}{L} \\
-\frac{1}{C_{1}} & 0 & 0 \\
\frac{1}{C_{2}} & 0 & 0
\end{array}\right], \\
& \mathbf{B}=\left[\begin{array}{cc}
0 & 0 \\
-\frac{1}{C_{1}} & 0 \\
0 & -\frac{1}{C_{2}}
\end{array}\right], \mathbf{C}=\left[\begin{array}{lll}
0 & 1 & 0 \\
0 & 0 & 1
\end{array}\right] .
\end{aligned}
$$

Here $i_{1}, v_{1}$ and $v_{2}$ are the state variable, $v_{1}$ and $v_{2}$ are also the output variables and $i_{s}, i_{l 1}$ and $i_{l 2}$ are the inputs of the system. $L=2 \cdot l_{\text {cable }} \cdot L_{\text {cable }}$ and $R=2 \cdot l_{\text {cable }} \cdot R_{\text {cable }}$ are the inductance and resistance of the cable, $l_{\text {cable }}$ is length of the cable, and $i_{s}, i_{l 1}$ and $i_{l 2}$ are currents flowing in the rectifier 


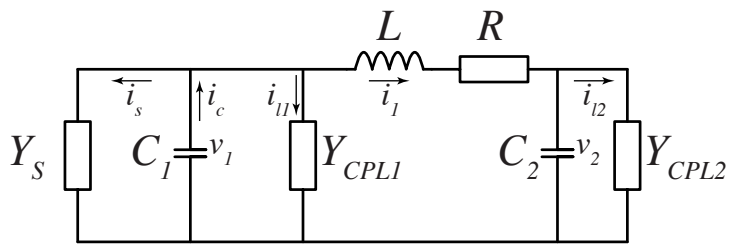

Fig. 4. Linearized model of the system given in Fig. 3(a).

and the two load converters, respectively.

$\mathbf{Y}(\mathrm{s})$ represents the active components of the systems in an admittance matrix. The main assumption to model the active components is to estimated the bandwidth for voltage (rectifier) and power (CPL motor drive) regulation. From [17], an IGCT based 3-L NPC (neutral point clamped) inverter with switching frequency $f_{s w}=250 \mathrm{~Hz}$ is considered; for this topology, a four time faster sampling frequency is reasonable to assume. It should be noted that this leads to a very limited control bandwidth compared to LV equipment where switching frequencies are around $10 \mathrm{kHz}$. Since voltage and power loops are outer ones, it is reasonable to set

$$
\alpha=0.08 \pi f_{s w}
$$

as the effective controller bandwdith. From this value, the gains of the system according to the model is section II.B are:

- The rectifier is modeled by $Y_{S}(s)$. The tuning is calculated by DVC rules [40] and considering the local capacitor as the physical plant, i.e., $K_{p}=\alpha C_{1}$.

- The CPL drives are modelled by $Y_{L 1}(s)$ and $Y_{L 2}(s)$. $P_{1}, P_{2}, V_{2}$ are taken from operation point of the drive. Their bandwidth is set by $\tau=0.1 / \alpha$, which set based on internal model control [43].

Finally, the resulting $\mathbf{Y}(\mathrm{s})$ matrix is a diagonal matrix and is given below:

$$
\mathbf{Y}(s)=\left[\begin{array}{cc}
Y_{S}(s)+Y_{L 1}(s) & 0 \\
0 & Y_{L 2}(s)
\end{array}\right]
$$

\section{B. Case 2 - Two Drives Connected Remotely}

The schematics of the passive components of this system are illustrated in Fig. 5. The variables defining the dynamics of this system are: (i) $i_{1}$ the current through the cable inductance connecting the first drive to the $C F U$, (ii) $i_{2}$ the current flows through the second cable connecting the second drive, (iii) $v_{1}$ the voltage of $C_{1}$, where $C_{1}=C F U+C_{c a b l e} / 2$, (iv) $v_{2}$ the voltage of $C_{2}$, where $C_{2}=C B U 1+C_{\text {cable }} / 4$, and (v) $v_{3}$ the voltage of $C_{3}$, where $C_{3}=C B U 2+C_{\text {cable }} / 4$. Here $L_{1}=$ $2 \cdot l_{\text {cable } 1} \cdot L_{\text {cable }}, L_{2}=2 \cdot l_{\text {cable } 2} \cdot L_{\text {cable }}, R_{1}=2 \cdot l_{\text {cable } 1}$. $R_{\text {cable }}$ and $R_{2}=2 \cdot l_{\text {cable } 2} \cdot R_{\text {cable }}$ are the inductances and resistances of the cables, $l_{\text {cable } 1}$ and $l_{\text {cable } 2}$ are the lengths of the two cables, and $i_{s}, i_{l 1}$ and $i_{l 2}$ are currents flowing in the rectifier and the two load converters, respectively. The statespace representation is given in Equation (11), where $i_{1}, i_{2}$, $v_{1}, v_{2}$ and $v_{3}$ are the state variables and $v_{1}, v_{2}$ and $v_{3}$ are also the output variables, and $i_{s}, i_{l 1}$ and $i_{l 2}$ are the inputs of the system:

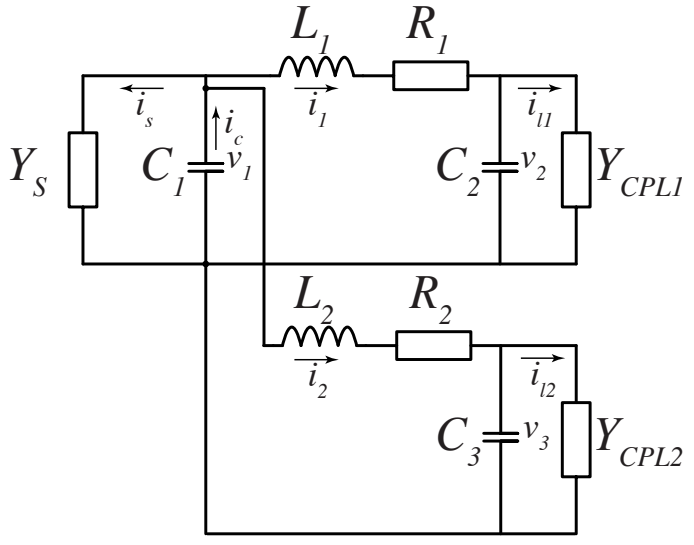

Fig. 5. Linearized model of the system given in Fig. 3(b).

$$
\begin{gathered}
x=\left[\begin{array}{l}
i_{1} \\
i_{2} \\
v_{1} \\
v_{2} \\
v_{3}
\end{array}\right], \mathbf{A}=\left[\begin{array}{ccccc}
-\frac{R_{1}}{L_{1}} & 0 & \frac{1}{L_{1}} & -\frac{1}{L_{1}} & 0 \\
0 & -\frac{R_{2}}{L_{2}} & \frac{1}{L_{2}} & 0 & -\frac{1}{L_{2}} \\
-\frac{1}{C_{1}} & -\frac{1}{C_{1}} & 0 & 0 & 0 \\
\frac{1}{C_{2}} & 0 & 0 & 0 & 0 \\
0 & \frac{1}{C_{3}} & 0 & 0 & 0
\end{array}\right], \\
\mathbf{B}=\left[\begin{array}{ccc}
0 & 0 & 0 \\
0 & 0 & 0 \\
-\frac{1}{C_{1}} & 0 & 0 \\
0 & -\frac{1}{C_{2}} & 0 \\
0 & 0 & -\frac{1}{C_{3}}
\end{array}\right], \mathbf{C}=\left[\begin{array}{lllll}
0 & 0 & 1 & 0 & 0 \\
0 & 0 & 0 & 1 & 0 \\
0 & 0 & 0 & 0 & 1
\end{array}\right] .
\end{gathered}
$$

The rectifier and inverter drive admittance components are the same as in Case 1, but with

$$
\mathbf{Y}(s)=\left[\begin{array}{ccc}
Y_{S}(s) & 0 & 0 \\
0 & Y_{L 1}(s) & 0 \\
0 & 0 & Y_{L 2}(s)
\end{array}\right]
$$

\section{Stability of the Representative MVDC DISTRIBUTION NETWORKS}

\section{A. Analysis and Discussion - Case 1}

Fig. 6 shows the eigenvalue movements for variations in $C B U 1, C B U 2$, and the distribution cable length as listed in Table II when the system is operating at its rated power. From Fig. 6(a), it can be observed that increasing CBU1 from $1 \mathrm{mF}$ to $20 \mathrm{mF}$ has a positive effect on the system stability and the eigenvalues move towards LHP. CBU1 directly impacts the tuning of the voltage controller of the rectifier and thus impacts stability the most. Additionally, it can be seen from Fig. 6(b) and Fig. 6(c) that the $C B U 2$ variations have minimal impact on the stability of the system for both stable and unstable system configurations. In Fig. 6(d) the impact of distribution cable is shown, and it can be observed that increasing cable lengths

TABLE II

PARAMETRIC VARIATIONS FOR STABILITY.

\begin{tabular}{|lr|lc|}
\hline CFU & $1-20 \mathrm{mF}$ & CBU1 & $1-20 \mathrm{mF}$ \\
CBU2 & $1-20 \mathrm{mF}$ & Cable Lengths & $1-5000 \mathrm{~m}$ \\
\hline
\end{tabular}




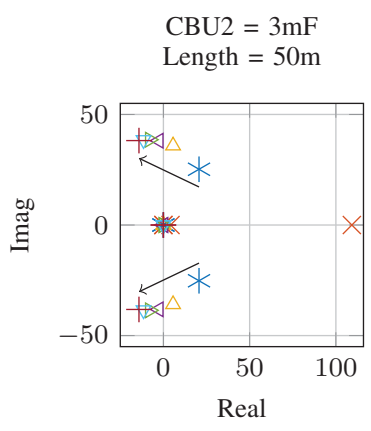

(a)

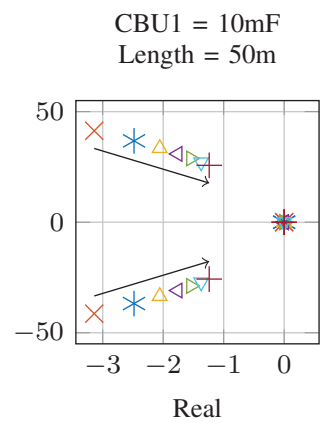

(b)

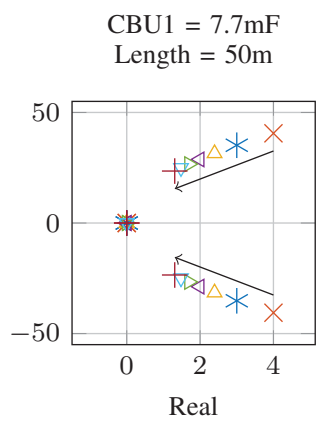

(c)

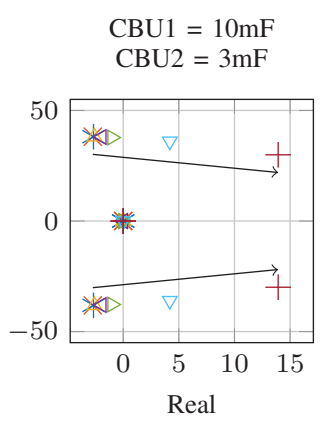

(d)

Fig. 6. Eigenvalue movements with changes in cable length, $\mathrm{CBU} 1$ and $C B U 2 . \times$ represents the lower value of the parameter, while + represents the highest value. The other symbols represents values in between. (a) $C B U 1$ variations with fixed $C B U 2$ and cable length. (b) $C B U 2$ variations with fixed $C B U 1$ and cable length for a stable configuration. (c) $C B U 2$ variations with fixed $C B U 1$ and cable length for an unstable configuration. (d) Distribution cable length variations with fixed $C B U 1$ and $C B U 2$.

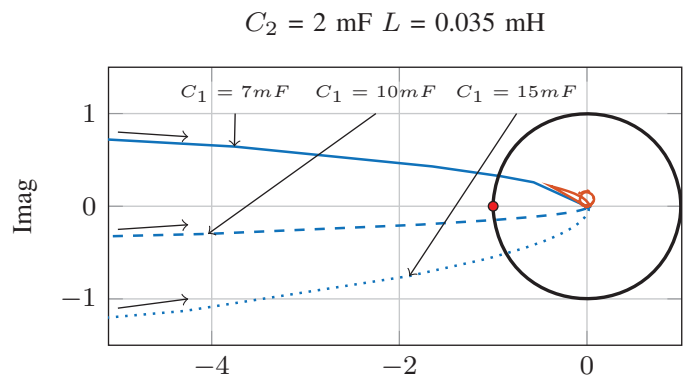

(a)

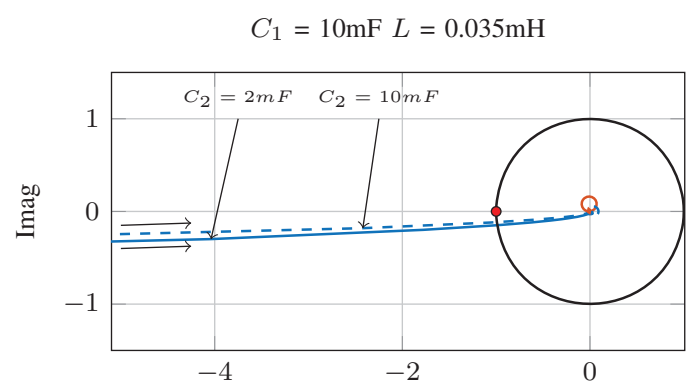

(b)

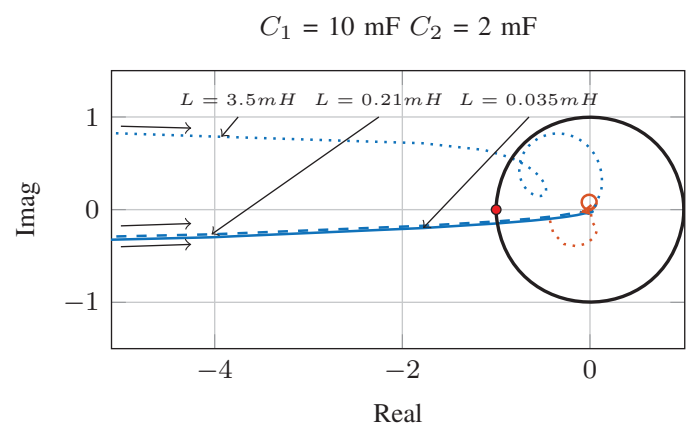

(c)

Fig. 7. Multi-variable Nyquist trajectories for case 1. The arrows on the figures show the direction of the trajectory. (a) Variations in CBUI (7 mF, $10 \mathrm{mF}, 15 \mathrm{mF})$. (b) Variations in $C B U 2(2 \mathrm{mF}, 10 \mathrm{mF})$. (c) Variations in distribution cable length $(10 \mathrm{~m}, 300 \mathrm{~m}, 5 \mathrm{~km})$. increases the distribution inductances in the system, therefore, causing instability in the system.

Fig. 7 alternatively shows the impact on the stability of the system in terms of Nyquist trajectories on complex plane. This representation is not influenced by static poles at the origin. As can be seen from Fig. 7, there are two eigenfunctions defining the dynamic behavior of case 1 . The blue trajectory represents the first eigenfunction $\lambda_{1}$ and it can be seen that it exits the unit circle, therefore, it can be considered the dominant eigenfunction. In order to declare a system absolutely unstable, the trajectories are expected to encircle the critical point of (1,0). However, from Fig. 7, it can be observed that there are no visual $(-1,0)$ encirclements. In such cases, the conformal mapping property can be considered to assess stability [44]. This property is briefly explained below.

From theory, any eigenvalue of the system, defined as $\lambda_{1}=$ $\sigma_{1}+j \omega_{1}$, should give a solution of the form

$$
\operatorname{eig}\left[\mathbf{Z}\left(s=\sigma_{1}+j \omega_{1}\right) \mathbf{Y}\left(s=\sigma_{1}+j \omega_{1}\right)\right]=-1+j 0
$$

The system is unstable if there is any eigenvalue for which $\sigma_{1}>0$. In practice, from an existing Nyquist plot, $\omega_{1}$ and $\sigma_{1}$ can be roughly estimated by inspection. First, a value for $\omega_{1}$ can be extracted by identifying the closest point of each Nyquist trajectory to -1 ; subsequently, the real part of the eigenvalue can be estimated by:

$$
\sigma_{1} \approx \Delta \omega \frac{\Lambda_{\Im, 1}\left(\omega_{1}\right)}{\Lambda_{\Re, 1}\left(\omega_{1}\right)-\Lambda_{\Re, 1}\left(\omega_{1}-\Delta \omega\right)}
$$

with $\Lambda(j \omega)$ representing the Nyquist trajectory in the polar plot and $\Delta \omega$ being a small frequency step between two consecutive points of the Nyquist trajectory. From (14), in practice, identification of the sign of $\sigma_{1}$ is straightforward by

TABLE III

STABLE CONFIGURATIONS FOR CASE 1.

\begin{tabular}{|l|c|}
\hline Parameter & Variations \\
\hline CBU1 & $8-20 \mathrm{mF}$ \\
CBU2 & $1-20 \mathrm{mF}$ \\
Cable Lengths & $1(0.693 \mu \mathrm{H})-300 \mathrm{~m}(0.208 \mathrm{mH})$ \\
\hline
\end{tabular}




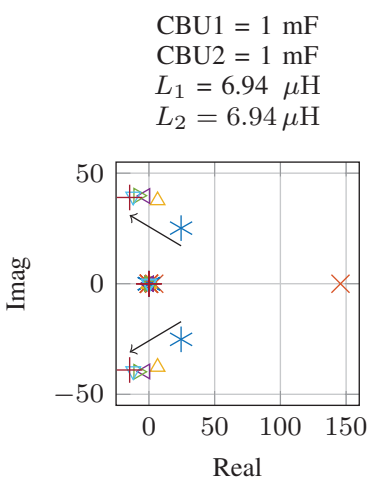

(a)

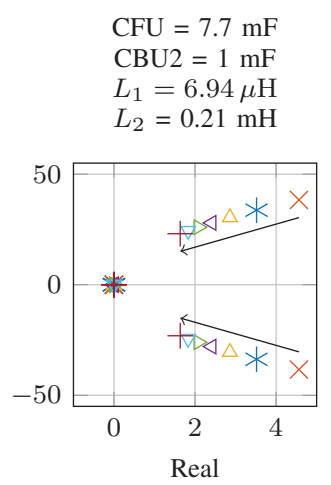

(b)

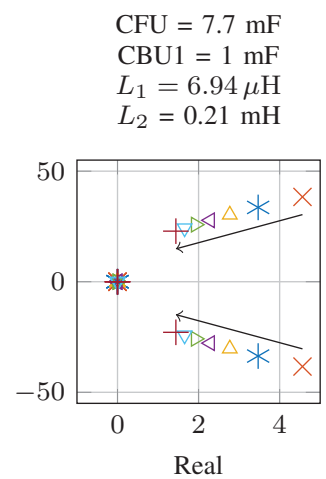

(c)

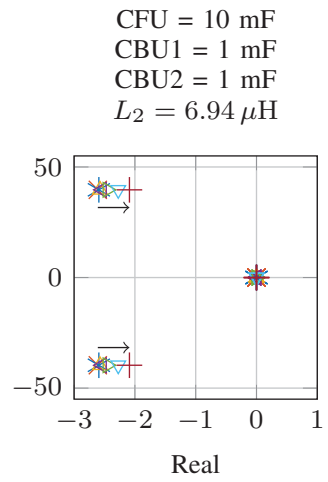

(d)

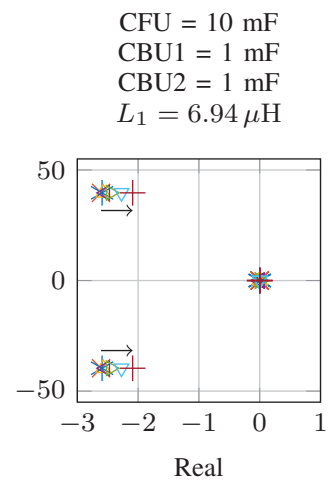

(e)

Fig. 8. Eigenvalue movements with changes in cable lengths, $C F U, C B U 1$ and $C B U 2 . \times$ represents the lower value of the parameter, while + represents the highest value. (a) $C F U$ variations with fixed $C B U 1, C B U 2$ and cable lengths at $10 \mathrm{~m}$. (b) $C B U 1$ variations with fixed $C F U, C B U 2$ and cable lengths at $10 \mathrm{~m}$ and $300 \mathrm{~m}$. (c) $C B U 2$ variations with fixed $C F U, C B U 1$ and cable lengths at $10 \mathrm{~m}$ and $300 \mathrm{~m}$. (d) and (e) variations in cable lengths from $10 \mathrm{~m}$ to $300 \mathrm{~m}$ with fixed $C F U, C B U 1$ and $C B U 2$.

inspection (of each Nyquist trajectory). More details, including a detailed derivation of (14), are given in [44].

Now, from Fig. 7(a), it can be observed that low value of $C B U 1$, i.e., $7 \mathrm{mF}, \Lambda_{1}$ lies in the II-quadrant and by applying Equation (14), it can be seen that the real part of eigenfunction is positive. This implies that the system is unstable for this system configuration. For bigger $C B U 1$ values, i.e., $10 \mathrm{mF}$ and $15 \mathrm{mF}$, the system becomes stable. From extension of this concept to Fig. 7(b) and Fig. 7(c), it can be observed that $C B U 2$ has minimum impact on the system stability, whereas, long distribution cable lengths, i.e., $5 \mathrm{~km}$ cause instabilities in the system.

Once the cable length is increased beyond $300 \mathrm{~m}$, the impact of $L$ and $C B U 2$ becomes more and more apparent in $\lambda_{2}$. It is observed that very high $L$ amplifies resonance in the system and higher $C B U 2$ is required to mitigate this instability. These impacts are further verified in time domain simulations.

One point to be noted here is that the very high capacitor sizes cannot be always used in the network because this might lead larger component space requirements, higher energy content (crucial during fault scenarios) and increased costs. Therefore, the system designer is always interested to know the minimum possible capacitance and inductance, which can guarantee proper operation with sufficient stability and lower overall costs.

\section{B. Analysis and Discussion - Case 2}

The dynamic analysis of the system is also computed for Fig. 3(b). In order to understand the impact of different passive elements on the system stability, at rated power operation, the parametric variations given in Table II are utilized. The pole movements directly resulting from these parametric variations are illustrated in Fig. 8. It can be seen from Fig. 8(a) that once again filter capacitance $C F U$, used for tuning the voltage control of the rectifier according to (9), plays a dominant role in the system stability $\left(C_{1} \approx C F U\right.$ in (9)). The other parameters, $C B U 1, C B U 2$ and cable lengths, have the least impact on the system stability. Similar conclusions can be drawn from Fig. 9. Here it can be observed that $C F U$ is the most critical among all the system parameters and directly impacts the ability of voltage control to damp the resonances

TABLE IV

STABLE CONFIGURATIONS FOR THE SYSTEM WITH TWO DRIVES CONNECTED REMOTELY.

\begin{tabular}{|l|c|}
\hline Parameter & Variations \\
\hline CFU & $10-20 \mathrm{mF}$ \\
CBU1 & $1-20 \mathrm{mF}$ \\
CBU2 & $1-20 \mathrm{mF}$ \\
Cable Lengths & $1-300 \mathrm{~m}$ \\
\hline
\end{tabular}




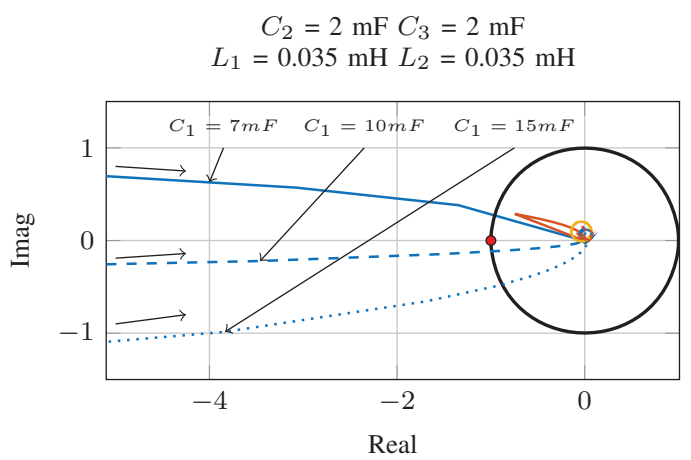

(a)

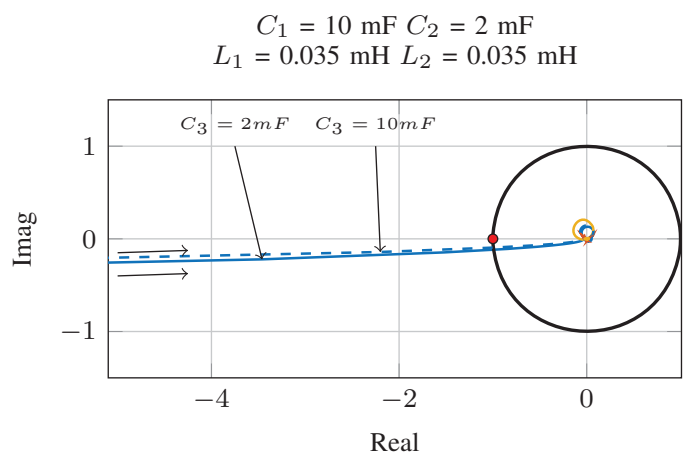

(c)

$$
\begin{gathered}
C_{1}=10 \mathrm{mF} C_{3}=2 \mathrm{mF} \\
L_{1}=0.035 \mathrm{mH} L_{2}=0.035 \mathrm{mH}
\end{gathered}
$$

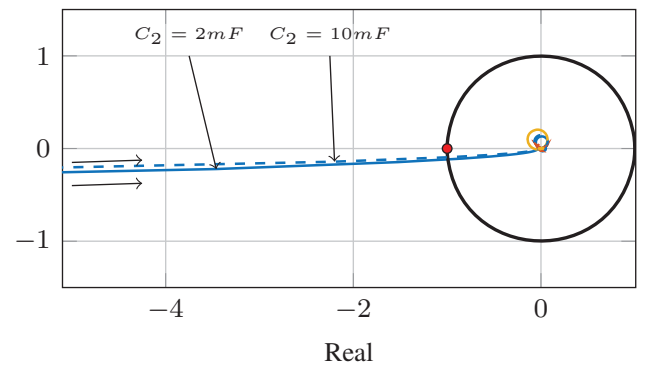

(b)

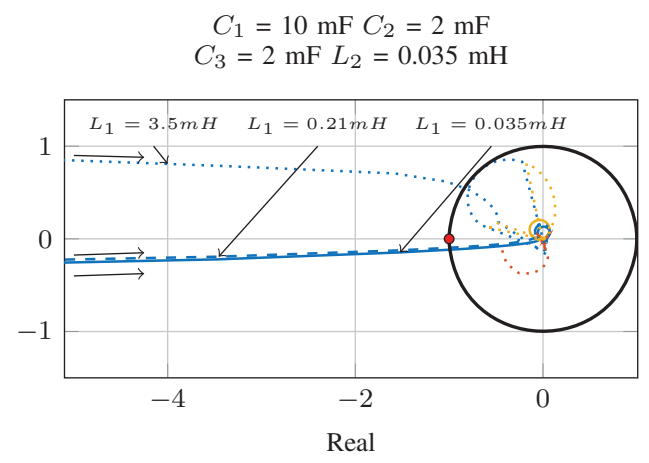

(d)

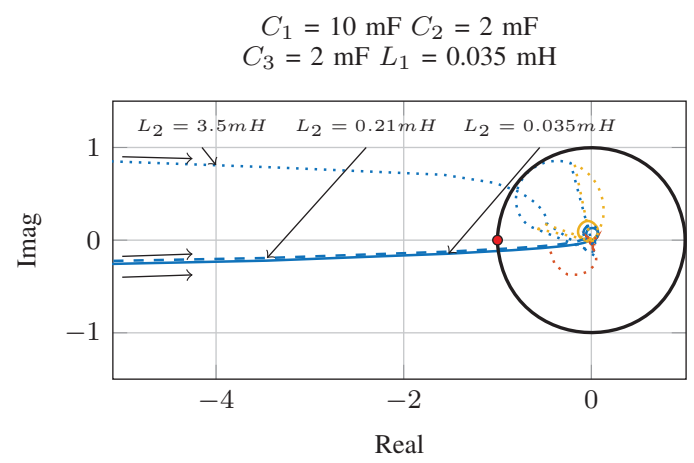

(e)

Fig. 9. Multi-variable Nyquist trajectories for case 2. The arrows on the figures show the direction of the trajectory. (a) Variations in $C F U$ ( $7 \mathrm{mF}, 10 \mathrm{mF}$ $15 \mathrm{mF})$. (b) Variations in CBUI $(2 \mathrm{mF}, 10 \mathrm{mF})$. (c) Variations in CBU2 (2 mF, $10 \mathrm{mF})$. (d) \& (e) Variations in distribution cable lengths (10 m, $300 \mathrm{~m}, 5 \mathrm{~km})$.

and maintain the dc-side voltage. The inverter filter capacitors $C B U 1$ and $C B U 2$ have minimal impact on stability. The distribution cables cause instability for long lengths, i.e., above $2 \mathrm{~km}$, as they introduce high inductance.

A summary of stable system configurations is given in Table IV, for cable lengths upto $300 \mathrm{~m}$ (300 m is chosen to emulate a reasonable large on-ship MVDC distribution networks). It can be seen that the impact of properly sized $C F U$ is required to maintain system stability. In this case, as both drives are fed remotely through cables, the voltage drop along these cables increases the load admittances and, therefore, a slightly higher $C F U$ is required to maintain system stability, for the same $\alpha$. Impact of high inductance of the cable is expected to be similar to the first case and is, therefore, not explored here.

\section{Full Model Time Domain Simulations}

The time domain simulations, using switching converter models for rectifier and inverters, for the systems shown in Fig. 3(a) and Fig. 3(b) are given in Fig. 10 and Fig. 11, respectively. These simulations are carried out in PLECS with complete cascaded control structures, considering switching and sampling delays, and filtering of reference values, implemented for both rectifier and inverter side. For the rectifier-side, as mentioned earlier, direct voltage control is implemented, where as for the inverter drives field oriented control is used. The control parameters are given in Table V. 
TABLE V

CONTROL PARAMETERS FOR SOURCE AND LOAD SUBSYSTEMS.

\begin{tabular}{|lclclclc|}
\hline$K_{p s, F O C}$ & $437500 \mathrm{~s}^{2} / \mathrm{kgm}^{2}$ & $K_{i s, F O C}$ & $13671875 \mathrm{~s}^{3} / \mathrm{kgm}^{2}$ & $K_{p i, F O C}$ & $0.338 \mathrm{~V} / \mathrm{A}$ & $K_{i i, F O C}$ & $3.456 \mathrm{Vs} / \mathrm{A}$ \\
$K_{p v, A R U}$ & $0.044 \mathrm{~A} / \mathrm{V}$ & $K_{i v, A R U}$ & $0.967 \mathrm{As} / \mathrm{V}$ & $K_{p i, A R U}$ & $0.5 \mathrm{~V} / \mathrm{A}$ & $K_{i i, A R U}$ & $4 \mathrm{e}-4 \mathrm{Vs} / \mathrm{A}$ \\
$K_{p, p l l, A R U}$ & $0.044 \mathrm{~s} / \mathrm{V}$ & $K_{i, p l l, A R U}$ & $987 \mathrm{~s} / \mathrm{V}$ & & & & \\
\hline
\end{tabular}

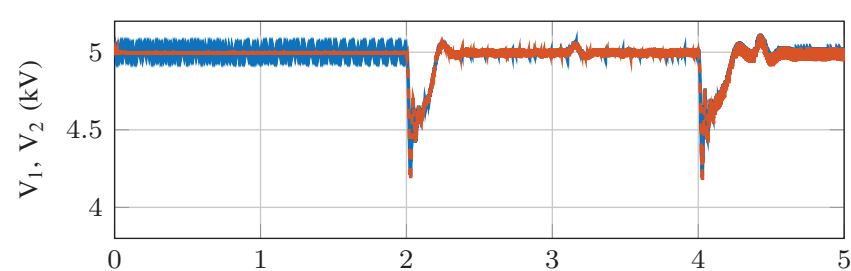

(a)

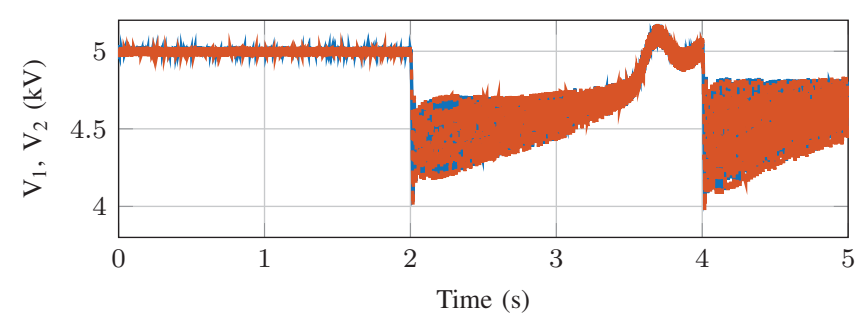

(c)

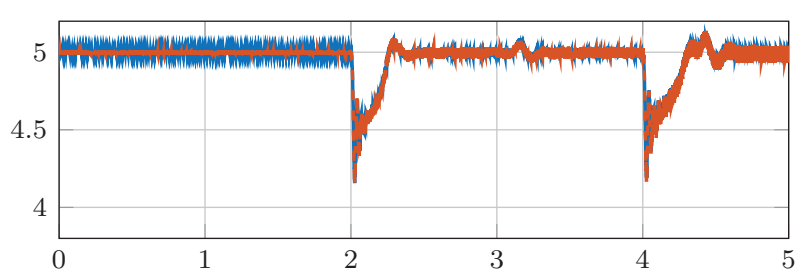

(b)

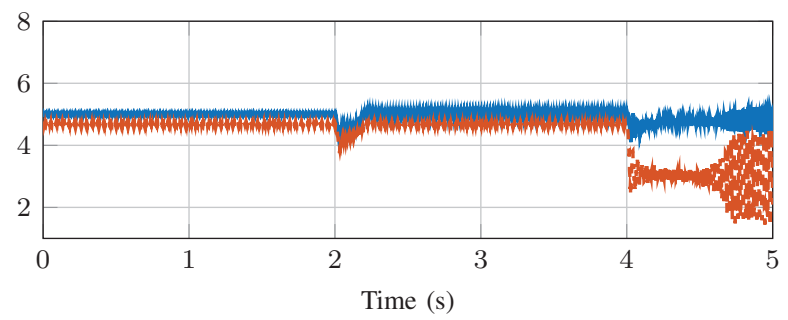

(d)

Fig. 10. Case 1: Fig. 3(a). Time domains simulations for load changes from $25 \%$ load to $62.5 \%$ at $2 s$ and $100 \%$ at $4 s$. Here $V_{1}-$ and $V_{2}---$ are the two variables presented. (a) $C B U 1=10 \mathrm{mF}, C B U 2=3 \mathrm{mF}$ and $l_{\text {cable }}=50 \mathrm{~m}$. (b) $C B U 1=7.7 \mathrm{mF}, C B U 2=3 \mathrm{mF}$ and $l_{\text {cable }}=50 \mathrm{~m}$. (c) $C B U 1=1 \mathrm{mF}$, $C B U 2=3 \mathrm{mF}$ and $l_{\text {cable }}=50 \mathrm{~m}$. (d) Impact of high cable inductance $C B U 1=10 \mathrm{mF}, C B U 2=3 \mathrm{mF}$ and $L_{\text {cable }}=2.5 \mathrm{mH}$.

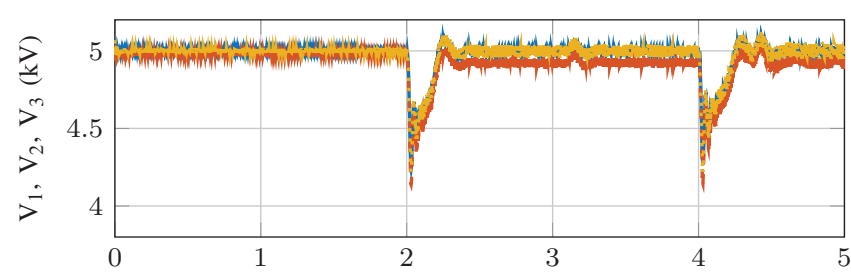

(a)

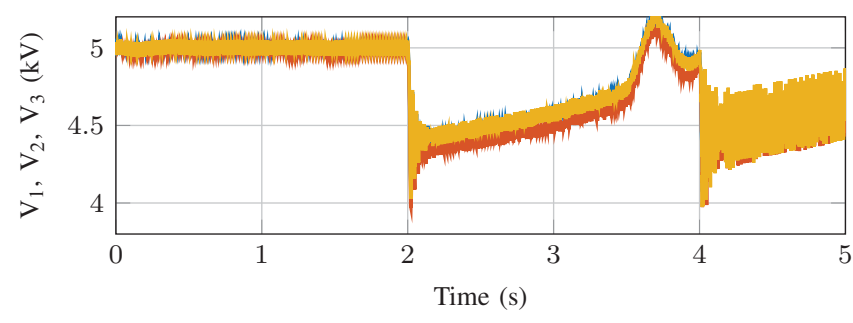

(c)

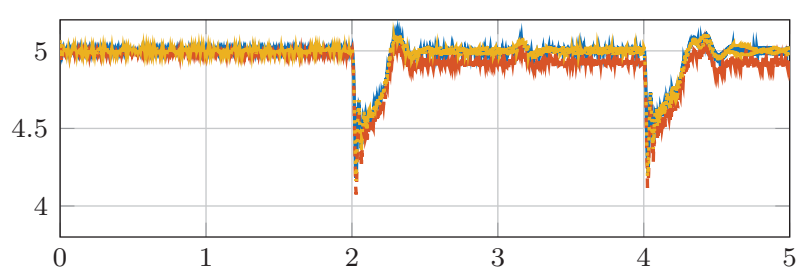

(b)

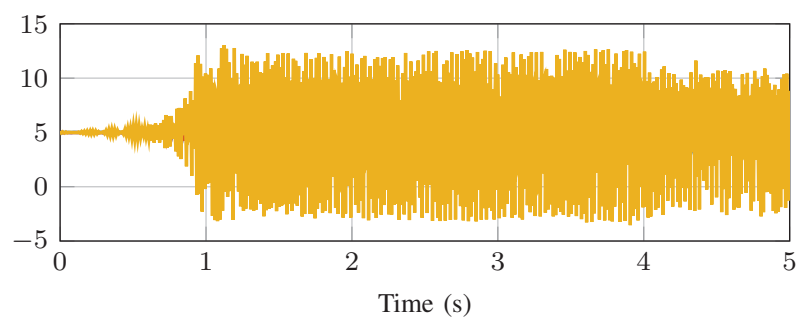

(d)

Fig. 11. Case 2: Fig. 3(b). Time domains simulations for load changes from $25 \%$ load to $62.5 \%$ at $2 \mathrm{~s}$ and $100 \%$ at $4 \mathrm{~s}$. Here $V_{1}-V_{2}---$ and $V_{3} \cdots \cdots$ are the three variables presented. (a) $C F U=10 \mathrm{mF}, C B U 1=3 \mathrm{mF}, C B U 2=3 \mathrm{mF}, l_{\text {cablel }}=300 \mathrm{~m}$ and $l_{\text {cable } 2}=300 \mathrm{~m}$. (b) $C F U=7.7 \mathrm{mF}, C B U 1=3 \mathrm{mF}, C B U 2$ $=3 \mathrm{mF}, l_{\text {cablel }}=300 \mathrm{~m}$ and $l_{\text {cable } 2}=300 \mathrm{~m}$. (c) $C F U=1 \mathrm{mF}, C B U 1=3 \mathrm{mF}, C B U 2=3 \mathrm{mF}, l_{\text {cablel }}=300 \mathrm{~m}$ and $l_{\text {cable } 2}=300 \mathrm{~m}$. (d) $C F U=10 \mathrm{mF}, C B U 1$ $=3 \mathrm{mF}, C B U 2=3 \mathrm{mF}, L_{\text {cable }}=0.21 \mathrm{mH}$ and $L_{\text {cable } 2}=3.5 \mathrm{mH}$.

Furthermore, the loading of the system is changed in steps from $25 \%$ to $62.5 \%$ at $2 \mathrm{~s}$ and finally to $100 \%$ at $4 \mathrm{~s}$.

These simulations are carried for different configurations. For case 1, it can be seen from Fig. 10 that higher $C B U 1$ results in stable response for different loading conditions, as can be observed from Fig. 10(a) where the system configuration is
$C B U 1=10 \mathrm{mF}, C B U 2=3 \mathrm{mF}$ and cable length is $50 \mathrm{~m}$. The system configuration of $C B U 1=7.7 \mathrm{mF}, C B U 2=3 \mathrm{mF}$ and $50 \mathrm{~m}$ cable length, with time domain results shown in Fig. 10(b), is expected to be unstable, from the analysis presented above, but it shows slightly longer settling times but stable behavior. One of the possible reasons is the repre- 

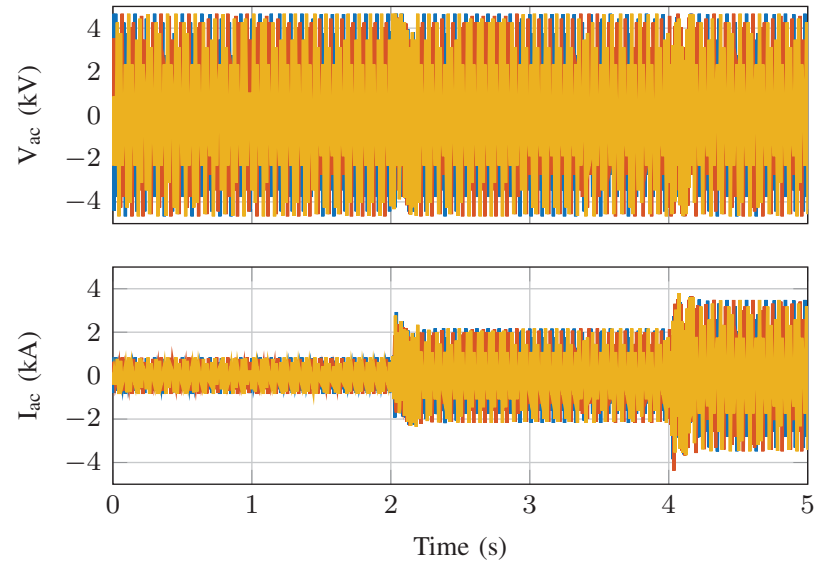

(a)
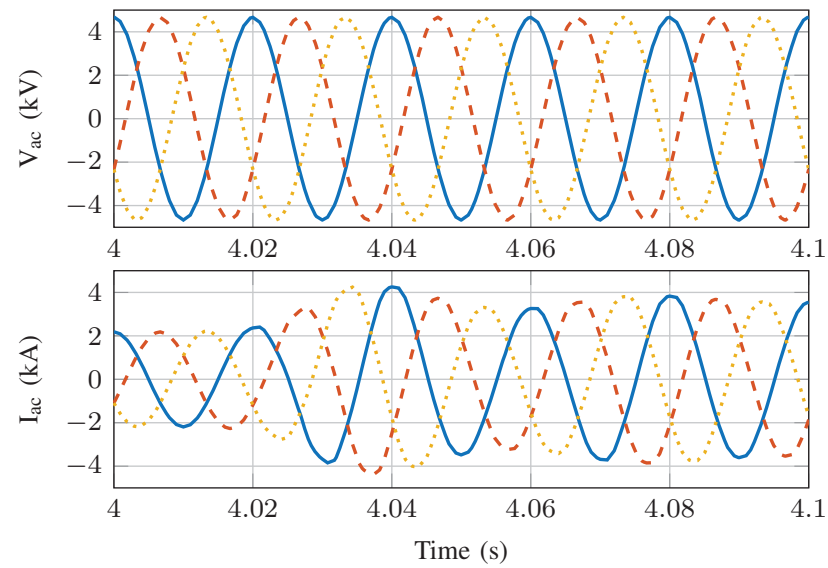

(c)
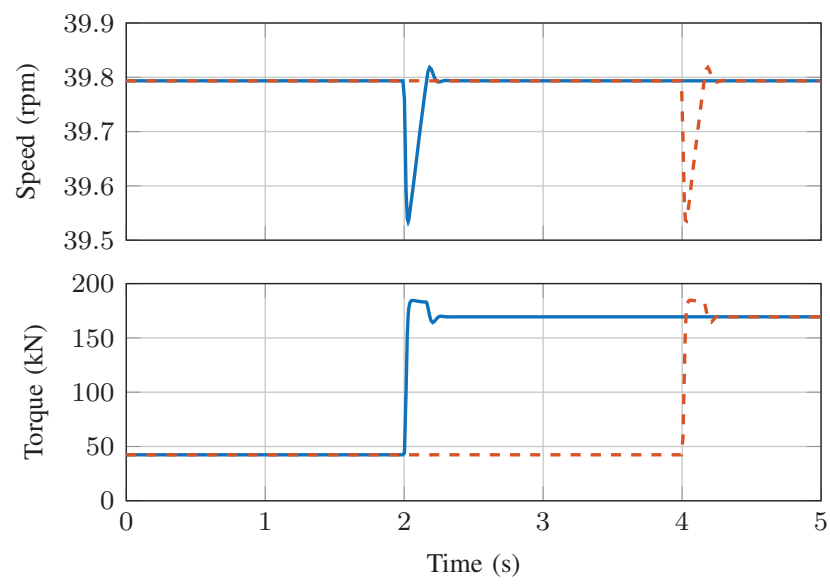

(e)
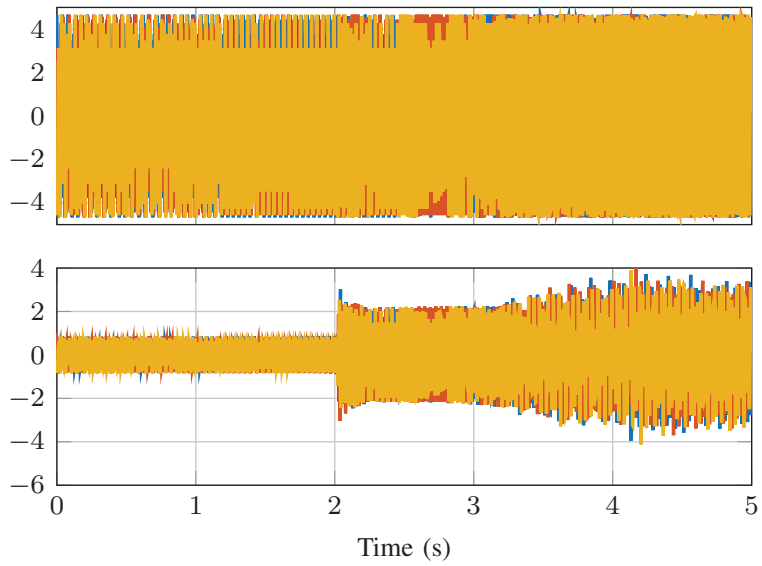

(b)
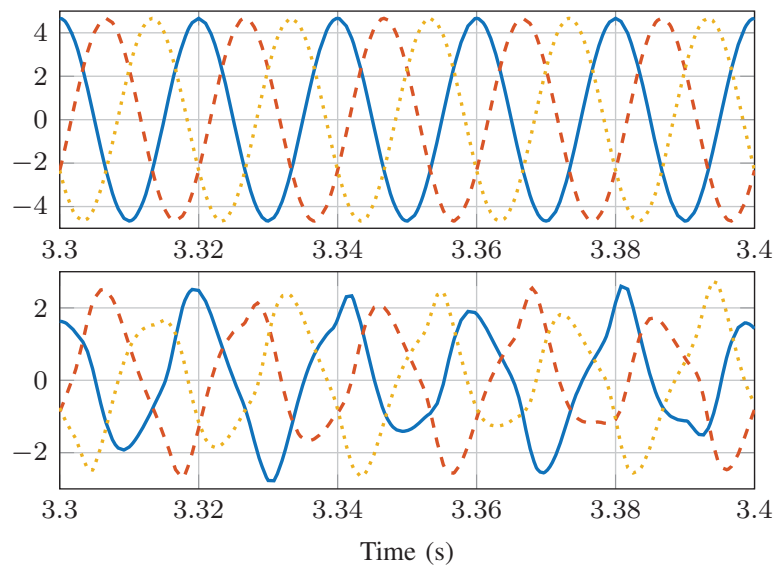

(d)
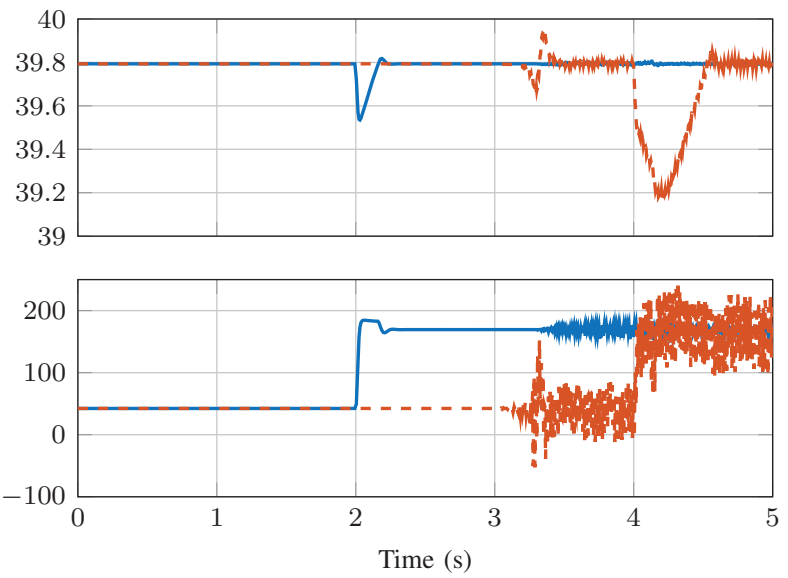

(f)

Fig. 12. AC-side voltage and currents (phase A - phase B - - -and phase $\mathrm{C}$..... (a) For stable system configuration with clearly increase in ac currents at $2 \mathrm{~s}$ and $4 \mathrm{~s}$ according to load changes. (b) For unstable system configuration with clearly increasing currents at $2 \mathrm{~s}$ for first load change that leads to system instability at $3 \mathrm{~s}$. (c) and (d) show zoom in plots of (a) and (b) highlighting the stable and unstable phenomena, respectively. (e) Changes in machine speeds and torque for stable system with load 1 changed at $2 \mathrm{~s}$ and load 2 - - -changed at $4 \mathrm{~s}$. (f) Changes in machine speeds and torque for unstable system configuration with load 1 changed at $2 \mathrm{~s}$ that leads to machine 2 speed drop at $3 \mathrm{~s}$ resulting in torque oscillations in load 2 . This worsens when the increase in load 2 at $4 \mathrm{~s}$.

sentation of the source admittance is not totally equal to the actual source admittance, due to the simplification of cascaded control and the stability is underestimated. Another possible reason is shift of unstable poles by the stiff solvers [45], [46], especially for configurations which are very close to stability/instability. 
The system configuration of $C B U 1=1 \mathrm{mF}, C B U 2=3 \mathrm{mF}$ and $50 \mathrm{~m}$ cable length, simulation shown in Fig. 10(c), is unstable as it has a large ripple and quite long response time compared to Fig. 10(a) and Fig. 10(b). Fig. 10(d) shows the time domain response for the system with high $L$, i.e., $C B U 1=10 \mathrm{mF}, C B U 2=3 \mathrm{mF}, L=2.5 \mathrm{mH}$ and $R=0.1 \mathrm{~m} \Omega$. Here, it can be observed that the system is unstable for very large $L$.

As the first drive is connected far from the rectifier, therefore, three voltages are observed in Fig. 11. Similar results to the first case, Fig. 3(a), are expected for Fig. 3(b) and can be seen in Fig. 11. Higher $C F U$ implies faster response of voltage control, while higher cable inductance leads to instability. The ac-side voltages and currents are shown in Fig. 12(a) and Fig. 12(c) for stable configurations (CFU $=10 \mathrm{mF}$ and small cable lengths $30 \mathrm{~m}$ ) for case 1 and 2 and Fig. 12(b) and Fig. 12(d) for unstable cases (CFU $=10 \mathrm{mF}$ and long cables 5 $\mathrm{km}$ ). Additionally, mechanical variables like speed and torque for both cases and stable and unstable configurations are shown in Fig. 12(e) and Fig. 12(f), respectively.

To summarize the analysis presented above, Fig. 7 and Fig. 9 show different values for capacitor banks and line inductances that can lead to instability. In that sense, this analysis fully provides a quantitative assessment of stability for each operation point. The corresponding time domain results in Fig. 10 and Fig. 11 illustrate the full load dynamics of the system for different configurations and cover the full range of possible system configurations. Full load dynamics are assumed to be critical operation points, since the constant power load behavior is maximized here [16], [17]. Therefore, by the proposed methodology and clear identification of critical operation points, a valuable understanding of system level stability/instability is given (e.g., reference [8] explicitly indicates that an identification of potential critical operation points should be performed as a part of the analysis).

\section{CONCLUSION}

In this work, multi-terminal MVDC distribution networks for electric ships, with special focus on available industrial technologies, are modeled and analyzed for their mutual dynamic interactions. These technologies are expected to be adopted for the earliest MVDC distribution networks for electric ships. MIMO impedance stability criterion has been employed in the analysis to map and understand the dynamic interaction among the sources and loads and also how do rectifier and inverter filters and cables lead to or can prevent systems from going towards instability. Two examples of the multi-terminal MVDC distribution networks with distributed layout are considered. The first example system considers two inverter drives supplied by a common source-side converter with one of them directly connected to the source, while the other is connected through a cable. In the second example, a generalized system is presented with both drives connected through cables.

From the MIMO stability analysis, it is found that the sizing of the system capacitances and their placement plays a significant role in the stability of the system. It is overall advantageous to place the higher capacitances close to the active rectifiers to improve the effective damping of the system (proportional gain of the voltage controller). Furthermore, a very high inductance, due to long cables or lossy cables, introduces low frequency resonances that can lead to system instability. All in all it can be seen that the present commercial technologies can be utilized for the initial deployment of MVDC distribution networks.

This work highlights importance of stability studies during system design phase, which can greatly increase assurance in reliability of the final system and the quality of operation. It must be pointed out here, this study does not lay steps to design an overall optimized system as that will be a multivariable problem (optimality will be subjective to many external factors surrounding any system) which is not the scope of this paper. In practical systems, a system designer or system integrator may not have access to relevant parameters of all the equipment and especially internal hardware or software details of power electronic converters, and parametric sweep studies, such as the one demonstrated in the paper, are of the utmost importance for designing a safe, reliable and cost effective system.

\section{REFERENCES}

[1] ABB AS. Onboard dc grid - the newest design for marine power and propulsion systems. (2012)

[2] Siemens Marine and Shipbuilding. BlueDrive PlusC - Makes vessels safer, more profitable and environmentally friendly. (2014)

[3] Rolls Royce. Tomorrow's ferry technology delivered today. (2016)

[4] J. F. Hansen, J. O. Lindtjørn, K. Vanska, and O. ABB, "Onboard de grid for enhanced dp operation in ships," in Dynamic Positioning Conference, 2011.

[5] N. H. Doerry and H. Fireman, "Designing all electric ships," in Proc. of the 9th Int. Marine Design Conf., pp. 475-498, 2006.

[6] G. Sulligoi, A. Tessarolo, V. Benucci, M. Baret, A. Rebora, and A. Taffone, "Modeling, simulation, and experimental validation of a generation system for medium-voltage dc integrated power systems," IEEE Trans. Ind. Appl., vol. 46, no. 4, pp. 1304-1310, Jul. 2010.

[7] U. Javaid, D. Dujic, and W. van der Merwe, "Mvdc marine electrical distribution: Are we ready?" in 41st Annu. Conf. of the IEEE Industrial Electronics Soc. (IECON), pp. 000 823-000 828, Nov. 2015.

[8] IEEE Recommended Practice for $1 \mathrm{kV}$ to $35 \mathrm{kV}$ Medium-Voltage DC Power Systems on Ships. IEEE Standard 1709, 2010.

[9] A. K. Aadnanes, "Maritime electrical installations and diesel electric propulsion,” Tech. Rep., 2003.

[10] A. Tessarolo, S. Castellan, R. Menis, and G. Sulligoi, "Electric generation technologies for all-electric ships with medium-voltage dc power distribution systems," in IEEE Electric Ship Technologies Symp. (ESTS), pp. 275-281, Apr. 2013.

[11] B. Zahedi and L. E. Norum, "Efficiency analysis of shipboard dc power systems," in 39th Annu. Conf. of the IEEE Industrial Electronics Soc. (IECON), pp. 689-694, Nov. 2013.

[12] M. S. Agamy, D. Dong, L. J. Garcés, Y. Zhang, M. E. Dame, X. Wu, and Y. Pan, "A high power medium voltage resonant dual active bridge for mvdc ship power networks," IEEE J. Emerg. Sel. Topics Power Electron., vol. 5, no. 1, pp. 88-99, Mar. 2017.

[13] R. Soman, M. M. Steurer, T. A. Toshon, M. O. Faruque, and R. M. Cuzner, "Size and weight computation of mvdc power equipment in architectures developed using the smart ship systems design environment," IEEE J. Emerg. Sel. Topics Power Electron., vol. 5, no. 1, pp. 40-50, Mar. 2017.

[14] G. O. Kalcon, G. P. Adam, O. Anaya-Lara, S. Lo, and K. Uhlen, "Smallsignal stability analysis of multi-terminal vsc-based dc transmission systems," IEEE Trans. Power Syst., vol. 27, no. 4, pp. 1818-1830, Nov. 2012.

[15] H. Suryanarayana and S. D. Sudhoff, "Design paradigm for power electronics-based DC distribution systems," IEEE J. Emerg. Sel. Topics Power Electron., vol. 5, no. 1, pp. 51-63, Mar. 2017. 
[16] A. Emadi, A. Khaligh, C. H. Rivetta, and G. A. Williamson, "Constan power loads and negative impedance instability in automotive systems: definition, modeling, stability, and control of power electronic converters and motor drives," IEEE Trans. Veh. Technol., vol. 55, no. 4, pp. 1112 1125, Jul. 2006.

[17] U. Javaid, F. D. Freijedo, D. Dujic, and W. van der Merwe, "Dynamic assessment of source-load interactions in marine mvdc distribution," IEEE Trans. Ind. Electron., vol. 64, no. 6, pp. 4372-4381, Jun. 2017.

[18] S. D. Sudhoff, S. F. Glover, P. T. Lamm, D. H. Schmucker, and D. E. Delisle, "Admittance space stability analysis of power electronic systems," IEEE Trans. Aerosp. Electron. Syst., vol. 36, no. 3, pp. 965 973, Jul. 2000.

[19] P. Liutanakul, A. B. Awan, S. Pierfederici, B. Nahid-Mobarakeh, and F. Meibody-Tabar, "Linear stabilization of a dc bus supplying a constant power load: A general design approach," IEEE Trans. Power Electron., vol. 25, no. 2, pp. 475-488, Feb. 2010.

[20] D. Dong, Y. Pan, R. Lai, X. Wu, and K. Weeber, "Active fault-curren foldback control in thyristor rectifier for dc shipboard electrical system," IEEE J. Emerg. Sel. Topics Power Electron., vol. 5, no. 1, pp. 203-212, Mar. 2017.

[21] S. Kim, D. Dujic, and S.-N. Kim, "Achieving protection selectivity in dc shipboard power systems employing additional bus capacitance," in Proc. of IECON, Washington DC, USA, 2018.

[22] A. Riccobono and E. Santi, "Comprehensive review of stability criteria for dc power distribution systems," IEEE Trans. Ind. Appl., vol. 50, no. 5, pp. 3525-3535, Sep. 2014.

[23] L. Guo, S. Zhang, X. Li, Y. W. Li, C. Wang, and Y. Feng, "Stability analysis and damping enhancement based on frequency-dependent virtual impedance for dc microgrids," IEEE Trans. Emerg. Sel. Topics Power Electron., vol. 5, no. 1, pp. 338-350, Mar. 2017.

[24] J. Sun, "Autonomous local control and stability analysis of multiterminal dc systems," IEEE J. Emerg. Sel. Topics Power Electron., vol. 3, no. 4 , pp. 1078-1089, Dec. 2015.

[25] X. Wang, Y. Peng, J. Zhu, Y. Xia, M. Yu, H. Hu, H. Cai, and W. Wei, "Decentralized impedance specifications for small-signal stability of dc distributed power systems," IEEE J. Emerg. Sel. Topics Power Electron., vol. 5, no. 4, pp. 1578-1588, Dec. 2017.

[26] A. M. I. Mohamad and Y. A. I. Mohamed, "Impedance-based analysis and stabilization of active dc distribution systems with positive feedback islanding detection schemes," IEEE Trans. Power Electron., vol. 33, no. 11 , pp. 9902-9922, Nov. 2018.

[27] A. Alacano, J. J. Valera, G. Abad, and P. Izurza, "Power-electronic-based dc distribution systems for electrically propelled vessels: A multivariable modeling approach for design and analysis," IEEE J. Emerg. Sel. Topics Power Electron., vol. 5, no. 4, pp. 1604-1620, Dec. 2017.

[28] M. K. Zadeh, R. Gavagsaz-Ghoachani, J. Martin, S. Pierfederici, B. Nahid-Mobarakeh, and M. Molinas, "Discrete-time tool for stability analysis of dc power electronics-based cascaded systems," IEEE Trans. Power Electron., vol. 32, no. 1, pp. 652-667, Jan. 2017

[29] G. Sulligoi, D. Bosich, G. Giadrossi, L. Zhu, M. Cupelli, and A. Monti, "Multiconverter medium voltage dc power systems on ships: Constantpower loads instability solution using linearization via state feedback control," IEEE Trans. Smart Grid, vol. 5, no. 5, pp. 2543-2552, Sep. 2014.

[30] D. Bosich, G. Giadrossi, and G. Sulligoi, "Voltage control solutions to face the cpl instability in mvdc shipboard power systems," in AEIT Annu. Conf. - From Research to Industry: The Need for a More Effective Technology Transfer, pp. 1-6, Sep. 2014.

[31] M. Cupelli, M. Moghimi, A. Riccobono, and A. Monti, "A comparison between synergetic control and feedback linearization for stabilizing mvdc microgrids with constant power load," in IEEE PES Innovative Smart Grid Tech., Europe, pp. 1-6, Oct. 2014.

[32] M. Cupelli, A. Monti, E. D. Din, and G. Sulligoi, "Case study of voltage control for mvdc microgrids with constant power loads comparison between centralized and decentralized control strategies,' in 18th Mediterranean Electrotechnical Conf. (MELECON), pp. 1-6, Apr. 2016.

[33] M. K. Zadeh, R. Gavagsaz-Ghoachani, S. Pierfederici, B. NahidMobarakeh, and M. Molinas, "Stability analysis and dynamic performance evaluation of a power electronics-based dc distribution system with active stabilizer,' IEEE J. Emerg. Sel. Topics Power Electron. vol. 4, no. 1, pp. 93-102, Mar. 2016.

[34] R. D. Middlebrook, "Input filter considerations in design and application of switching regulators," IAS Record, 1976.

[35] X. Wang and F. Blaabjerg, "Harmonic stability in power electronic based power systems: Concept, modeling, and analysis," IEEE Trans. Smart Grid, vol. to be published, 2018 .
[36] C. Zhang, X. Cai, A. Rygg, and M. Molinas, "Sequence domain SISO equivalent models of a grid-tied voltage source converter system for small-signal stability analysis," IEEE Trans. Energy Convers., vol. 33, no. 2, pp. 741-749, Jun. 2018.

[37] M. Molinas, "Impedance-based stability evaluation of power electronics dominated power systems," in IEEE PELS-IES Delhi Chapter Expert Lecture, 2019.

[38] M. Zubiaga, A. Cárcar, G. Abad, J. A. Barrena, and S. Aurtenetxea, Evaluation of the Frequency Response of AC Transmission Based Offshore Wind Farms. INTECH Open Access Publisher, 2011.

[39] J. Arocas-Pérez and R. Griño, "A local stability condition for dc grids with constant power loads," IFAC-PapersOnline, vol. 50, no. 1, pp. 7-12, Jul. 2017.

[40] L. Harnefors, M. Bongiorno, and S. Lundberg, "Input-admittance calculation and shaping for controlled voltage-source converters," IEEE Trans. Ind. Electron., vol. 54, no. 6, pp. 3323-3334, Dec. 2007.

[41] G. C. Goodwin, S. F. Graebe, and M. E. Salgado, Control System Design. Prentice Hall, 2000.

[42] M. Amin and M. Molinas, "Small-signal stability assessment of power electronics based power systems: A discussion of impedance- and eigenvalue-based methods," IEEE Trans. Ind. Appl., vol. 53, no. 5, pp. 5014-5030, Sep. 2017

[43] L. Harnefors and H. P. Nee, "Model-based current control of ac machines using the internal model control method," IEEE Trans. Ind. Appl., vol. 34, no. 1, pp. 133-141, Jan. 1998.

[44] F. D. Freijedo, U. Javaid, and D. Dujic, "Conformal mapping of impedance stability models for system-level dynamics assessments," in International Symposium on Power Electronics (Ee), pp. 1-6, Oct. 2017.

[45] F. E. Cellier and E. Kofman, Continuous system simulation. Springer Science \& Business Media, 2006

[46] PLECS, "An introduction to modeling and simulation using plecs," Aalborg, Denmark, 2015.

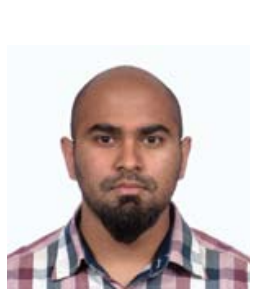

Uzair Javaid (S'14-M'18) received his B.Sc. in Electrical Engineering, in 2010, from University of Engineering and Technology, Lahore. He obtained his M.Sc. in Energy Management and Sustainability and Ph.D. in Electrical Engineering from Ecole Polytechnique Federale de Lausanne (EPFL) in 2013 and 2018, respectively. Since May 2018, he is working as a system engineer for Grids and Power Quality Solutions, ABB Switzerland. His research interests include MVDC distribution systems, flexible AC transmission systems, power electronic system modeling and design and electrical machine drives.

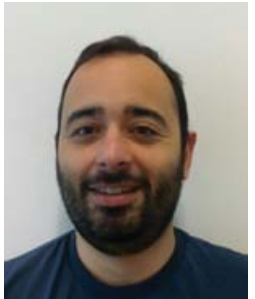

Francisco D. Freijedo (M'07-SM'16) received the M.Sc. degree in physics from the University of Santiago de Compostela, Santiago de Compostela Spain, in 2002 and the Ph.D. degree in electrical engineering from the University of Vigo, Vigo, Spain, in 2009. From 2005 to 2011 , he was a Lecturer in the Department of Electronics Technology, University of Vigo. From 2011 to 2014, he worked with Gamesa Innovation and Technology as a Power Electronics Control Engineer for renewable energy applications. From 2014 to 2016, he was a Postdoctoral Researcher in the Department of Energy Technology, Aalborg University. From 2016 to 2019, he was a Scientific Collaborator of the Power Electronics Laboratory, Ecole Polytechnique Federale de Lausanne. Currently, he is working in Industry as a Power Electronics Control Expert. The main research interests are devoted to power conversion technologies. Dr. Freijedo is an Associate Editor for the IEEE TRANSACTIONS ON INDUSTRIAL ELECTRONICS

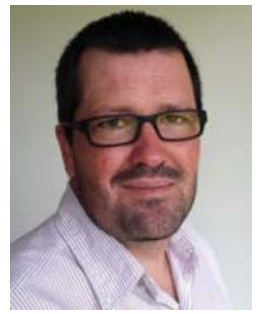

Wim van der Merwe (M'03-SM'14) received the B.Eng., M.Sc. (Eng), and Ph.D. degrees, in electrical engineering from the University of Stellenbosch, Stellenbosch, South Africa, in 2000, 2006, and 2011, respectively. He joined ABB Corporate Research in 2012 and is currently R\& D Platform Manager of the ACS6000 MV-drive at ABB Medium Voltage Drives in Turgi, Switzerland. His research interests include multileveland medium-voltage power electronic converters and the modeling of complex power electronic systems. 


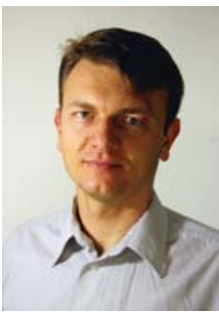

Drazen Dujic (S'03-M'09-SM'12) received the Dipl.-Ing. and M.Sc. degrees from the University of Novi Sad, Novi Sad, Serbia, in 2002 and 2005, respectively, and the Ph.D. degree from the Liverpool John Moores University, Liverpool, U.K., in 2008. From 2002 to 2006, he was with the Department of Electrical Engineering, University of Novi Sad as a Research Assistant, and from 2006 to 2009 with Liverpool John Moores University as a Research Associate. From 2009 till 2013, he was with ABB Corporate Research Centre, Switzerland as a Principal Scientist working on the power electronics projects spanning the range from low-voltage/power SMPS in below kilowatt range to medium voltage high-power converters in megawatt range. From 2013 till 2014, he was with ABB Medium Voltage Drives, Turgi, Switzerland, as R\& D Platform Manager. He is currently with Ecole Polytechnique Federale de Lausanne EPFL, Lausanne, Switzerland, as an Assistant Professor and the Director of the Power Electronics Laboratory. His current research interests include the areas of design and control of advanced high-power medium-voltage power electronics conversion systems and applications. He has authored or co-authored more than 100 scientific publications and has filed 12 patents. $\mathrm{He}$ is an Associate Editor for IEEE Transactions on Industrial Electronics, IEEE Transaction on Power Electronics and IET Electric Power Applications. In 2018, he received EPE Outstanding Service Award and in 2014, the Isao Takahashi Power Electronics Award for outstanding achievement in power electronics. 